\title{
Characterization of the Chemistry and Structure of Material Transfer on AFM Tips Resulting from Contact and Sliding Experiments
}

\author{
C. J. Tourek, S. Sundararajan \\ * Department of Mechanical Engineering, Iowa State University, Ames, IA 50011
}

Atomic force microscopy (AFM) is used extensively in the fields of nanoscience and nanotechnology to investigate tribomechanical phenomena on the micro and nano scales. While AFM based techniques have proven very useful in investigating nanotribological phenomena, a detailed assessment of the material structure and chemistry of the near apex region of the AFM tip can provide further insights. Specific areas that would directly benefit from this information include fabrication and friction/wear studies using AFM tips where material transfer and tip chemistry are of importance [1-4]. Few techniques exist that can provide this critical information. We recently reported that the material structure and chemistry of the near apex regions of commercial AFM tips can be successfully interrogated using atom probe tomography (APT) down to the near atomic-scale [5]. APT is a technique capable of concurrently determining three dimensional material structure and chemistry at near atomic resolution which has seen expanding use in science and engineering fields $[6,7]$. Typical AFM tips are commercially available with a conical tip with a tip radius less than $100 \mathrm{~nm}$ that makes them suitable for analysis by atom probe tomography (APT), and by utilizing an appropriate holder, an AFM tip can be analyzed using APT and 3D reconstructions of the atom positions in the near apex region of the AFM tip can be obtained. We present our investigations here utilizing a Local Electrode Atom Probe (LEAP) to study the formation of transferred material during dry sliding experiments involving a commercially available $\mathrm{Si}$ AFM tip on $\mathrm{Cu}$. In Figure $1 \mathrm{Cu}$ is present in the mass spectrum obtained from APT on an AFM tip after dry sliding on $\mathrm{Cu}$. The presence of $\mathrm{Cu}$ demonstrates that $\mathrm{Cu}$ is transferring to the AFM tip. Also, the well defined sharp peaks of $\mathrm{Si}$ and $\mathrm{Cu}$ in the mass spectrum indicate that the $\mathrm{Cu}$ adheres to the silicon AFM tip strongly enough to field evaporate in a controlled manner. 3D reconstructions are used to measure the thickness of the transferred $\mathrm{Cu}$ layer, and investigate diffusion of the $\mathrm{Cu}$ into the $\mathrm{Si}$. The effects of sliding distance and normal load on the formation of transferred material are also reported.

\section{References}

[1] K.H. Chung, D.E. Kim, Ultramicroscopy, 108 (2007) 1-10.

[2] K.S.K. Karuppiah et al., Tribology Letters, 36 (2009) 259-267.

[3] K.S.K. Karuppiah et al., Langmuir, 25 (2009) 12114-12119.

[4] K. Tanaka et al., Journal of Nanomaterials, (2009).

[5] C. Tourek, S. Sundararajan, Microsc. Microanal., 16 (2010) 636-642 .

[6] T.F. Kelly, M.K. Miller, Review of Scientific Instruments, 78 (2007) 20.

[7] D.N. Seidman, Ann. Rev. Mater. Res., 37 (2007) 127-158.

[8] This research was supported by a grants from the National Science Foundation (Grant no. 0932573), the W. M Keck Foundation, and Iowa State University. 

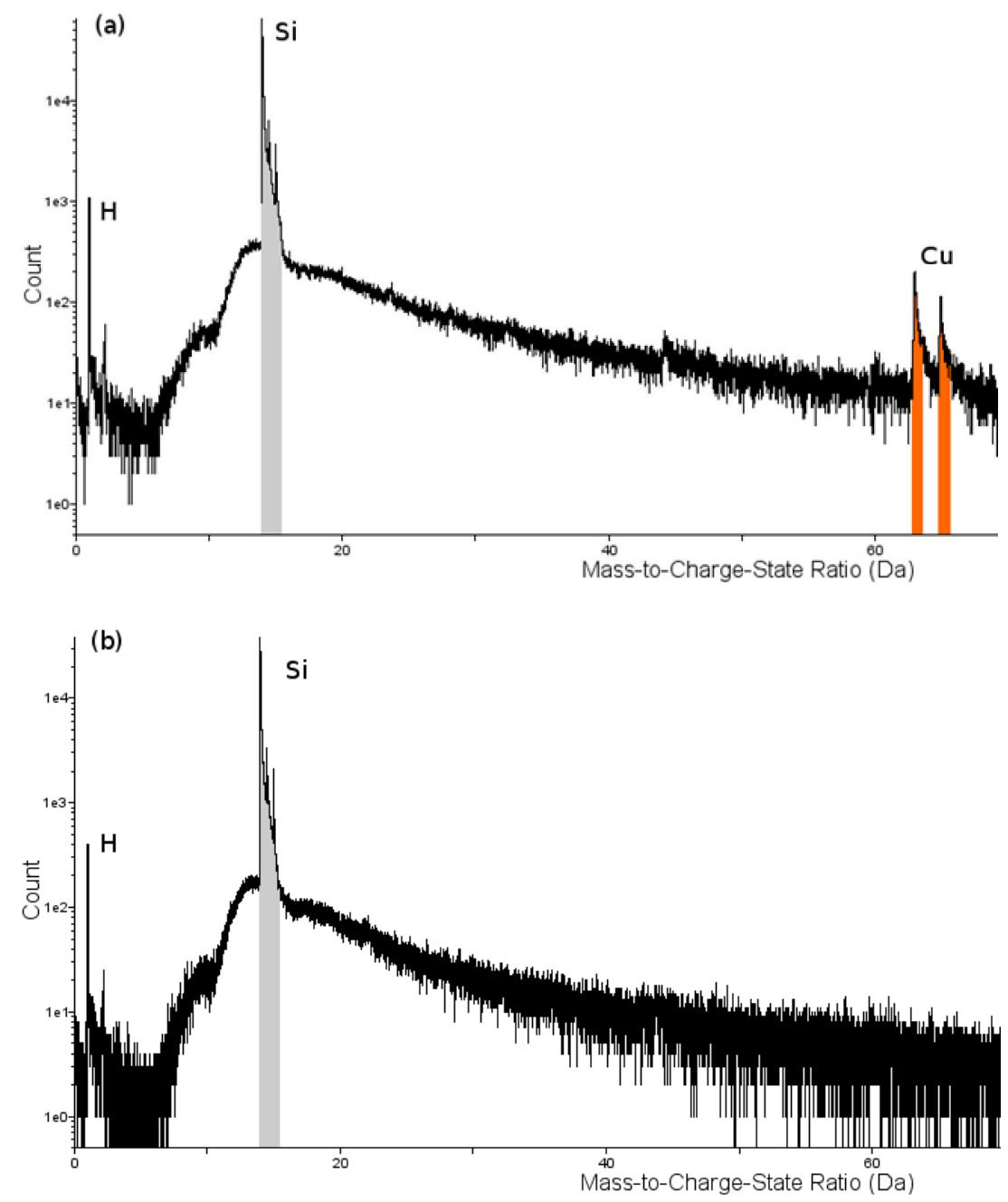

FIG. 1. Typical mass spectrum from an APT run on (a) an AFM tip used in a dry sliding experiment on $\mathrm{Cu}$ showing the presence of $\mathrm{Cu}$ and (b) an unused AFM tip for comparison. 